\title{
Forum
}

\section{Les nouveaux liens sociaux au territoire}

\author{
Christian Deverre \\ Sociologue, INRA Écodéveloppement, site Agroparc, Domaine Saint-Paul, 84914 Avignon cedex 9, France
}

\author{
Mots-clés : \\ territoire rural ; \\ lien social ; \\ modernisation \\ agricole ; \\ environnement ; \\ agronomie
}

\section{Keywords:} rural territory; social tie; agricultural modernisation; environment; agronomy

\begin{abstract}
Résumé - L'essor des préoccupations concernant les effets des activités agricoles sur la qualité de l'environnement remet en cause le mouvement de spécialisation spatiale et sociale établi par le processus de modernisation agricole. Basé sur l'objectif de maximisation de la production alimentaire, ce processus a été soutenu par d'importants aménagements structurels des territoires (irrigation, drainage, remembrements) et par une législation qui a assuré la prééminence des droits des producteurs agricoles sur l'espace rural au détriment des autres usagers. Dans les domaines scientifique et technique, l'agronomie et l'économie rurale ont joué un rôle dominant dans l'établissement de cette différenciation spatiale et sociale. Les préoccupations environnementales remettent en cause à la fois la monofonctionnalité de l'usage des territoires et leur appropriation prioritaire par les producteurs agricoles. La multifonctionnalité des territoires s'accompagne de l'établissement de nouveaux systèmes de relations entre agriculteurs et autres usagers de l'espace rural. Dans cette conjoncture, l'agronomie doit aussi composer avec d'autres disciplines scientifiques, comme l'écologie, dans l'orientation des techniques agricoles. La remise en cause du processus de spécialisation productive spatiale et sociale donne de manière croissante aux territoires ruraux le statut de biens publics, mais les forces du marché peuvent également entraîner une nouvelle tendance à la spécialisation et à la privatisation environnementales.
\end{abstract}

\begin{abstract}
The new social links to territories. The growing of concerns about the effects of agricultural activities on the quality of environment questions the trend towards social and spatial specialisation established by the process of agricultural modernisation. This process aimed at the maximisation of food production. It was supported by important territorial planning and infrastructures (irrigation systems, land reclamation, land consolidation) and by laws which provided farmers with pre-eminent rights of access to rural space to the detriment of other potential users. In the scientific and technical fields, agronomy and rural economy played a dominant part in establishing this spatial and social specialisation. Environmental concerns question both the monofunctionality of territorial use and the priority of farmers' access rights. The multifunctionality of territories goes with the institutionalisation of new relation systems between farmers and other rural space stakeholders. In these circumstances, agronomy has to come to terms with other scientific disciplines, such as ecology, in the field of technical recommendations. This questioning of productive spatial and social specialisation increasingly provides rural territories with the status of public goods. But market forces can also lead to new environmental specialisation and land privatisation.
\end{abstract}

J'ai pour habitude, dans la relecture de mes écrits, de faire la chasse au terme «nouveau». Outre le fait que ce que l'on baptise souvent hâtivement «nouveau» se révèle, à un examen plus approfondi, pas si nouveau que cela, l'usage de ce mot est également un moyen commode de ne pas nommer plus substantiellement l'objet ou le phénomène analysé. Et voici que le titre que j'ai moi-même choisi pour cet article inclut le terme banni. La raison de cette rupture de tabou tient à l'embarras que j'éprouve à caractériser par un (ou des) nom(s) précis la forme (ou les formes) des liens sociaux

Auteur correspondant : deverre@avignon.inra.fr qui m'apparai(ssen)t émerger entre différents groupes d'agents autour des territoires ruraux, notamment à l'occasion de l'essor des politiques environnementales ${ }^{1}$.

Pour expliquer cet embarras, reprenons les fondamentaux. Pour Émile Durkheim (1930), le passage de la solidarité mécanique qui caractérise les sociétés archaïques à la solidarité organique des sociétés modernes fut le produit de la différenciation des individus qui

\footnotetext{
${ }^{1}$ Ce texte est issu des $2^{\mathrm{e}}$ Entretiens du Pradel, «Agronomes et territoires» (12 et 13 septembre 2002). Une première version a été publiée sur le site de l'Académie d'agriculture (http://www.academie-agriculture.fr/publications/colloques).
} 
elle-même résulte de la division du travail social. C'est la reconnaissance mutuelle de rôles et de fonctions différents et complémentaires à l'intérieur du système social qui fonde les liens sociaux de la solidarité organique. Chaque rôle et chaque fonction sont régis par un système de droits et d'obligations envers les autres agents, droits et obligations relevant pour Durkheim de formes de droit restitutif, en opposition au droit répressif des liens sociaux de la similitude, la solidarité mécanique. Le mouvement de division du travail social s'accentue à mesure que la société se densifie (accroissement du nombre des individus et des échanges entre eux) et l'expansion corrélative $\mathrm{du}$ nombre de règles de droit restitutif ou coopératif (civil, commercial, administratif...) peut être considérée comme un indicateur du progrès.

Au niveau spatial, la différenciation des individus et la division du travail social se traduisent par le principe de mise à distance, principe énoncé par Georg Simmel (1981) et étudié empiriquement par l'écologie urbaine de l'école de Chicago (Grafmeyer et Joseph, 1990). L'espace de la solidarité organique est en conséquence lui aussi fragmenté, individualisé. Ses territoires se spécialisent, sans pour autant s'autonomiser : suivant le même modèle que les règles de droit et d'obligations entre agents, la circulation des personnes, des biens et de l'argent y est intense, régie par chacune des fonctionnalités reconnues aux différentes fractions de l'espace.

Pour évolutionniste qu'elle soit, cette représentation de l'évolution des liens sociaux dans les sociétés modernes et ses implications territoriales rendent assez bien compte de l'évolution des activités agricoles et des territoires ruraux dans la période dite de la modernisation. J'y reviendrai dans un moment.

Néanmoins, depuis quelques années, l'image se brouille, le modèle paraît s'emmêler. De la pollution à la "malbouffe", en passant par la destruction des milieux, les activités agricoles sont soumises à de profondes critiques qui remettent en cause, en grande partie, le système de droits et d'obligations des agriculteurs établi à l'époque des Trente Glorieuses (Deverre, 1995). On assiste à la multiplication des dispositions juridiques d'encadrement des pratiques et techniques au titre de justifications diverses, de la sécurité alimentaire à la protection de la biodiversité, des eaux ou contre les risques. Ces justifications sont portées par des groupes et agents eux aussi très divers qui revendiquent la redéfinition des obligations des détenteurs de droits sur les différentes fractions de l'espace rural. Ceci rend de plus en plus difficile à saisir le rôle ou la fonction attendue par la société de ses agriculteurs. La fortune du terme «multifonctionnalité » en témoigne.

En ce qui concerne les territoires ruraux, le nombre croissant de zonages superposés répondant à ces justifications conduit à s'interroger sur le mouvement de mise à distance : tout se passe comme si l'empilement succédait à la juxtaposition, et c'est avec bonheur que les systèmes d'informations géographiques remplacent les cartes à une seule dimension.

Comment donc caractériser les liens sociaux qui s'établissent dans ce contexte autrement que par la pauvreté de l'adjectif «nouveaux»?

\section{Le dispositif de la modernisation agricole}

Pour tenter d'apporter des éléments de réponse à cette question, il m'apparaît utile de faire un retour sur la période dite de modernisation agricole, et d'utiliser comme grille de lecture de ce mouvement les notions de « dispositif » et de «bio-politique » développées par Michel Foucault.

La notion de dispositif, même si elle est présente dans de nombreuses œuvres de Michel Foucault, n'est vraiment théorisée que dans ses derniers travaux, en particulier dans le premier tome de son Histoire de la sexualité (1976). Pour lui, la nature du dispositif est d'être « un ensemble résolument hétérogène, comportant des discours, des institutions, des aménagements architecturaux, des décisions réglementaires, des lois, des mesures administratives, des énoncés scientifiques, des propositions philosophiques, morales, philanthropiques [...]. Le dispositif lui-même, c'est le réseau que 1'on peut établir entre ces éléments » (Foucault, 1994, p. 299). Cet ensemble organisé d'éléments hétérogènes constitue « une formation qui, à un moment historique donné, a eu pour fonction majeure de répondre à une urgence. Le dispositif a donc une fonction stratégique dominante » (ibid.).

Le processus de modernisation de l'agriculture française au lendemain de la Seconde Guerre mondiale (et même quelques années auparavant) peut être appréhendé globalement au travers de cette notion de dispositif. À l'époque, l'urgence stratégique dominante est de fournir en toute sécurité une alimentation abondante et bon marché à une population ouvrière en expansion mise au service de la reconquête de la puissance nationale (Chombart de Lauwe, 1979). L'agriculture métropolitaine - mais aussi coloniale - se voit alors complètement remodelée par la conjonction organisée d'un ensemble impressionnant de ces éléments hétérogènes dont parle Michel Foucault. Ces éléments sont suffisamment connus pour ne pas les reprendre en détail (Gervais et al., 1976) et je n'examinerai ici que deux catégories avant de revenir sur la nature des liens sociaux établis par cette période de modernisation agricole : les « aménagements architecturaux » et les "énoncés scientifiques ».

Si géographes et historiens ont depuis longtemps insisté sur le fait que les activités humaines, et en particulier les activités agricoles, ont constamment modelé et remodelé les paysages (Dion, 1991; Pitte, 1989), il est incontestable que les « équipements architecturaux » 
du dispositif de modernisation de l'agriculture ont été colossaux. On peut citer à cet égard les nombreuses opérations de remembrement, le développement des infrastructures d'irrigation et de drainage ou les entreprises, à grande échelle, d'amendement des sols "pauvres ». La levée des contraintes - le terme est fréquemment utilisé à cette époque - imposées par l'histoire agraire, le climat ou les milieux entraîne une redéfinition radicale de la différenciation territoriale. En conséquence, les cartographies des pédologues ne se contentent plus de dresser l'inventaire des types de sols - ce qui conduisait à identifier des terroirs ayant telle ou telle vocation -; elles parlent désormais de potentialités agricoles, c'est-à-dire des utilisations possibles des sols après aménagement. Le sec peut devenir humide grâce à la Compagnie du BasRhône ou l'Office du Niger, l'humide peut devenir sec par les équipements de drainage appropriés. Certes, certaines données du milieu ne peuvent être aussi commodément corrigées, comme l'altitude : on parlera alors de handicaps, appelant des mesures spéciales, un traitement à part.

Je vais retenir ce couple potentialités-handicaps pour introduire une autre notion empruntée à Michel Foucault, celle de bio-politique. Par ce terme, il désigne l'ensemble des entreprises d'optimisation du fonctionnement des populations (humaines) de l'époque moderne, de la médecine publique aux grands travaux hygiénistes, en passant par la résorption et/ou la mise à l'écart des catégories sociales pourvues de handicaps, comme les fous, les délinquants ou les pauvres.

Si les dispositifs caractérisant la bio-politique n'excluent pas la répression - notamment par le biais de l'enfermement (Foucault, 1975) -, ils apparaissent plus souvent sous la forme de techniques positives du pouvoir, visant à assurer les meilleures conditions de croissance et de productivité des populations. Elles s'appuient, pour ce faire, sur le développement de la rationalité et sur des connaissances scientifiques et techniques (en particulier, dans les cas étudiés par Michel Foucault, sur la médecine et l'économie politique).

L'objectif stratégique du dispositif de la modernisation agricole, centré sur la sécurité de l'approvisionnement alimentaire de la classe ouvrière, s'intègre bien dans les orientations de la bio-politique. Et ses effets territoriaux, au travers des aménagements architecturaux, peuvent être considérés comme une prise sur le sol par des groupes d'experts scientifiques et techniques (le génie rural) comparable à la prise sur les corps de la médecine ou sur les esprits de la psychiatrie. Déjà, au XIX ${ }^{\mathrm{e}}$ siècle, les travaux hygiénistes avaient donné lieu à de telles prises sur les éléments physiques, comme l'endiguement des fleuves pour éviter la propagation des miasmes des eaux vagabondes (Foucault, 1994, pp. 207-228).
Dans cette prise sur le sol à finalité de production agricole et sur les agents qui l'utilisent, deux disciplines scientifiques ont joué un rôle important par la production d'énoncés normatifs qui ont donné aux aménagements architecturaux leur efficacité économique et sociale : l'agronomie, dans sa définition largo sensu, et l'économie rurale.

Par l'élaboration de modèles raisonnés associant les propriétés pédo-climatiques des parcelles agricoles, leurs améliorations et amendements potentiels, les choix de cultures et de variétés végétales ou animales adéquats, les formes de travail du sol et les successions appropriées, les agronomes contribuèrent à proposer des itinéraires techniques pour optimiser les rendements des productions. Les économistes ruraux, souvent issus des mêmes formations que les précédents, explorèrent les espaces de validité de ces modèles en fonction de l'organisation qu'ils impliquaient, des facteurs de production et des substitutions possibles entre eux. Croisés avec les données des rendements et de l'état des marchés existants ou souhaitables - et un niveau de revenu standard jugé équitable pour un ménage agricole ${ }^{2}$, ces travaux permirent, pour chaque type de système de culture (ou d'élevage) et chaque zone pédo-climatique adéquate, de définir la structure optimale (superficie, parcellaire, équipement. ..) des «exploitations agricoles », terme qui remplace la ferme d'antan.

Sans être imposés par voie d'obligation, par droit répressif, ces énoncés scientifiques, ces modèles normatifs de l'agronomie et de l'économie rurale furent traduits et diffusés au travers de l'appareil parapublic de formation, de vulgarisation et de conseil qui se met également en place à l'époque. Et toute une série de dispositions légales et administratives les reprennent pour sélectionner parmi les aspirants agriculteurs ceux qui peuvent bénéficier des soutiens publics en matière $d^{\prime}$ 'investissement et $\mathrm{d}$ 'accès au foncier.

Ainsi schématiquement - et seulement partiellement - résumé, le dispositif de modernisation de l'agriculture a contribué à modeler le système des droits et obligations des différents groupes participant à sa finalité stratégique, à commencer par celui des agriculteurs. Ceux parmi les producteurs de produits agricoles qui acceptèrent de participer activement à l'entreprise d'optimisation de l'usage des sols se virent reconnaître les droits d'accès au soutien public, que ce soutien se traduise par des flux financiers, des ressources humaines ou des prééminences foncières.

Ces droits fonciers peuvent être caractérisés par la mise à distance des détenteurs passés ou potentiels

\footnotetext{
${ }^{2}$ Ce choix de l'agriculture familiale, qui donnera naissance à la fameuse "exploitation agricole à 2 UTH (unités de travail humain) ", tire sa source pour sa part des "propositions philosophiques, morales, philanthropiques » du dispositif de modernisation que nous n'abordons pas ici.
} 
d'autres droits, à commencer par les propriétaires : le statut du fermage, le contrôle des sociétés d'aménagement foncier et d'équipement rural (SAFER), les modalités d'accès aux prêts fonciers bonifiés, les catégories des Plans d'occupation des sols, tout l'arsenal d'administration des terres agricoles a tendu à écarter des décisions les concernant, ceux qui ne participaient pas à l'entreprise d'optimisation. Même si elles s'avérèrent parfois plus conflictuelles, les opérations de remembrement consacrèrent également la priorité de l'obligation productive sur les droits des propriétaires minoritaires et des riverains. Seuls les usages liés aux besoins du développement industriel et urbain (infrastructures, lotissements...) prenaient le pas sur cette prééminence agricole.

Il faut cependant souligner que cette mise à distance des autres groupes sociaux de l'usage des terres désignées comme étant à finalité agricole n'est accordée aux agriculteurs qu'au prix de leur insertion affirmée dans le mouvement de la division du travail social. Les engrais, les semences (végétales ou animales), les machines, le capital, proviennent de l'extérieur de l'exploitation; la spécialisation réduit comme peau de chagrin la part de l'autoconsommation; les savoirs et les savoir-faire sont partagés avec l'appareil d'accompagnement; jusqu'à la comptabilité des ménages qui est parfois confiée aux centres de gestion. La part des produits commercialisés destinée à l'industrie et aux grands distributeurs allant croissant, les contrats d'intégration limitent l'activité commerciale propre des agriculteurs. On est bien là dans le double mouvement de spécialisation territorialeau sens physique comme social - et d'approfondissement de la division sociale du travail.

\section{L'émergence des dispositifs des politiques environnementales dans l'espace rural}

Il semble que l'on assiste dorénavant à un renversement de ce double mouvement. Au niveau territorial, la prééminence de l'usage productif des terres agricoles est de plus en plus fortement contestée au titre des «externalités »-comme le disent les économistes pour lesquels le paradigme de la maximisation reste central auxquelles les pratiques d'optimisation des rendements ont conduit. Que ce soit dans le domaine de la qualité des eaux ou de la protection de la biodiversité, pour prendre ces exemples, de nouveaux territoires sont configurés, qui réduisent ou gomment la distance établie précédemment entre terres agricoles et non agricoles. Le bassin versant ou le biotope d'une population d'oiseaux réunissent terres arables, prairies, forêts, réserves naturelles, mais aussi espace bâti, routes et autres réseaux...

Et dans ce mouvement de réduction de la distance, les agriculteurs sont de manière croissante appelés à réintégrer dans leurs systèmes techniques des éléments
" externalisés », dont l'administration avait été attribuée à d'autres agents dans le cadre de la division du travail social. Les distributeurs d'eau potable, dont les équipements d'épuration n'arrivent plus à satisfaire les normes publiques, exigent la réduction des flux d'intrants, le recyclage des déchets agricoles ou d'élevage au sein des exploitations. Les collectivités locales, lasses d'endiguer les rivières, demandent la reconversion de terres drainées en prairies humides. Des conservatoires naturels, impuissants sur leurs seules réserves à maintenir les espèces dont ils ont pris la charge, poussent à la réimplantation des haies et à la reconstitution des bocages balayés par les remembrements. Et je ne ferai ici que mention des autres pressions qui, au titre cette fois de la sécurité sanitaire des aliments, réclament la réduction des traitements pesticides, le développement de techniques de lutte intégrée.

Toutes ces pressions sur les activités agricoles, qui remettent en cause les cloisonnements territoriaux, poussent donc aussi à la réintégration au sein de l'exploitation d'éléments du travail social auparavant dévolus à d'autres. Peut-on dans ces conditions considérer que «l'internalisation» des conséquences environnementales de l'activité agricole remet en cause, au moins partiellement, le processus de division du travail social (Deverre et al., 2002) ? Et dans ce cas, comment qualifier l'évolution concomitante des liens sociaux entre les différents agents et groupes intervenant sur les territoires ruraux?

Lorsque l'on observe ce qui se passe sur le terrain, on se trouve face à des mouvements paradoxaux. D'un côté en effet, la maîtrise par les agriculteurs d'impacts environnementaux de leurs pratiques peut les conduire à s'autonomiser - au moins relativement - d'un certain nombre d'autres agents, notamment des firmes d'approvisionnements en intrants ou en aliments de bétail, des agences d'équipement hydraulique... Mais dans le même temps, l'adoption des nouvelles pratiques est accompagnée, surveillée, évaluée par de nouveaux agents au travers des nombreux «comités de pilotage » qui les entourent. Je ne prendrai comme exemple que l'entrée d'associations environnementalistes ou de consommateurs dans les commissions départementales d'orientation agricole chargées de l'approbation et du suivi des contrats territoriaux d'exploitation ou maintenant des contrats d'agriculture durable.

Mais pour prendre toute la mesure de cette évolution paradoxale, il me paraît une nouvelle fois utile de faire appel à la notion de dispositif, et d'inventorier l'ensemble des éléments hétérogènes qui forment les réseaux des politiques environnementales dans l'espace rural, et de qualifier la ou les fonction(s) stratégique(s) dominante(s) qu'elles sont amenées à remplir.

Cette identification des fonctions stratégiques dominantes, si elle ne pose pas de problème particulier quand on isole tel ou tel objectif environnemental, 
en pose beaucoup plus si l'on se pose globalement le problème et que $l^{\prime}$ on émet l'hypothèse $\mathrm{d}$ 'une convergence de l'ensemble des objectifs des politiques sectorielles autour de notions du type du « développement durable». J'avais déjà abordé ces questions il y a quelques années (Deverre, 1995) au travers de la métaphore de l'archipel. La multiplication des îles environnementales (la qualité des eaux, la protection de la biodiversité, la prévention de tel ou tel risque naturel...) peut-elle être considérée comme un continent en formation ou n'est-elle que la manifestation de hot spots, de points chauds localisés et restant séparés, aussi nombreux qu'ils puissent être?

Il ne m'appartient pas d'apporter moi-même une réponse à cette question, mais de la chercher dans l'examen de la mise en œuvre des politiques environnementales. Or, force est de constater que, au-delà des discours unificateurs organisés autour du développement durable ou de la multifonctionnalité, la sectorialisation reste largement la règle et que la convergence n'apparaît pas comme l'hypothèse la plus envisageable à moyen terme. J'en prendrai pour exemple le récent Schéma de services collectifs des espaces naturels et ruraux (Datar, 2000) qui additionne plusieurs dizaines de fonctions associées à autant d'objectifs et faisant chacune l'objet d'une cartographie propre. Aucune réponse n'y est vraiment apportée au fait que ces fonctions et objectifs puissent se recouper partiellement sur un même espace. Le responsable d'un parc naturel régional évoquait récemment devant moi le sentiment que l'ensemble dont il avait la charge se trouvait littéralement « haché » par les divers dispositifs des politiques territoriales.

Certes, chacune des fonctions stratégiques des divers dispositifs des politiques environnementales peut être pensée dans la perspective d'une extension de la biopolitique à de nouveaux domaines et à de nouveaux sujets, mais le projet d'ensemble n'est pas visible, la portée de chacun d'eux est différente. Certains, comme la potabilité des eaux, ressortent de l'hygiénisme classique, tandis que d'autres, notamment lorsqu'ils prennent en considération des justifications bio- ou éco-centriques (Larrère et Larrère, 1997) constituent une extension de la biopolitique de l'optimisation des populations humaines vers celle du vivant en général, voire au-delà lorsque l'on revendique de "penser comme un fleuve » ou «penser comme une montagne » (Leopold, 1995, pp. 168-172). Chacun des réseaux d'éléments hétérogènes qui constituent les différents dispositifs est en partie imperméable aux autres et il n'est pas possible de superposer l'organisation des valeurs, connaissances, institutions, décisions et propositions morales, disons, de l'administration d'une zone vulnérable ou d'un site Natura 2000.

Avant de revenir sur les conséquences de cette pluralité de dispositifs émergents sur les liens sociaux autour des territoires ruraux, je voudrais évoquer ma perception de la place de l'agronomie dans cette configuration contemporaine. De nombreux agronomes ont perçu les enjeux, pour leur discipline, que constitue la prise en compte des conséquences environnementales des actes techniques de conduite des cultures et, sans cacher les difficultés, considèrent avec optimisme ses capacités à y apporter des réponses, notamment sur la base de son ouverture pluridisciplinaire et de ses acquis en matière de modélisation systémique (Benoît et Papy, 1998; Lemaire et Meynard, 1998 ; Papy, 2001).

J'aurai pour ma part quelques réserves à apporter à cet optimisme. Le problème n'est en effet pas seulement d'apporter des réponses pertinentes à des enjeux identifiés, mais d'insérer les énoncés scientifiques produits dans les dispositifs prenant en charge ces enjeux. Or, plusieurs éléments me paraissent assez radicalement différents dans le contexte actuel par rapport à celui de la modernisation agricole. Je ne reprendrai ici que ceux que j'ai introduits dans l'ébauche d'analyse de ce dispositif de modernisation.

L'étroite association de l'agronomie et de l'économie rurale ne paraît plus aller de soi. Outre le fait qu'ils sont maintenant issus de formations différentes de celles des agronomes, la manière dont les économistes traitent les questions d'environnement, même s'ils le font parfois à reculons, comme en témoigne la notion d'externalité, peut les amener à être plus rétifs à s'approprier les modèles agronomiques. Si l'optimum de Pareto accordait assez aisément la maximisation des rendements et la maximisation du bien-être général, cette dernière peut dorénavant contrarier la première - conduisant par exemple à l'introduction du principe pollueur-payeur -, voire à l'éliminer, comme dans le cas où la valeur accordée à un milieu peu ou pas anthropisé serait supérieure à celle de la production agricole. Â contrario, le coût de la réduction de certains impacts environnementaux envisagés par le génie agronomique pourrait s'avérer trop élevé par rapport aux pertes productives.

Par ailleurs, l'articulation entre les éléments architecturaux des dispositifs des politiques environnementales et les énoncés scientifiques agronomiques ne va pas de soi non plus. La traduction du biotope d'une espèce ou du bassin versant $d$ 'une rivière en objets faisant sens pour la science qui étudie «la conduite des champs cultivés » (Lemaire et Meynard, 1998) s'annonce plus incommode que celle d'un périmètre irrigué ou d'une commune remembrée construits précisément pour améliorer les conditions de la production agricole. Outre le fait que les échelles territoriales ne sont plus les mêmes, qu'elles ne coïncident plus avec des entités faisant sens pour l'agronome comme pour l'agriculteur, et qu'elles intègrent bien d'autres éléments que les champs cultivés, les objets mêmes que ces dispositifs identifient comme pivots sont au moins périphériques, sinon complètement étrangers à ceux de l'agronomie, comme les bords de champs, les fossés, les arbres isolés, les souches ou les broussailles. 
Enfin, pour participer à un réseau, encore faut-il y être invité, et il n'est pas certain que les agronomes et leurs connaissances soient mobilisés dans tous les dispositifs impliquant les activités agricoles. Comme mentionné plus haut, rien n'indique que la sectorialisation des politiques environnementales serait dépassée et que l'intégration systémique des objectifs productifs et environnementaux soit la priorité de chacune d'elles. Certes, les premières politiques agri-environnementales ont largement recyclé des éléments du dispositif de la modernisation, mais certains signes indiquent que toutes celles qui suivent, et suivront, ne feront pas de même. D'autres centres d'expertise, articulés à d'autres communautés et disciplines scientifiques, affirment qu'ils peuvent concurrencer la place centrale de l'agronomie, y compris dans ses champs d'excellence. Une enquête en cours sur les ressources mobilisées par les opérateurs chargés d'élaborer les documents d'objectifs sur les sites de la directive Habitats en France fait ainsi apparaître des organismes comme l'Atelier technique des espaces naturels ou Espaces naturels de France comme des centres d'expertise non seulement des milieux naturels, mais aussi des mesures de gestion des territoires. Autre exemple, même s'il peut apparaître un peu anecdotique : le programme du colloque sur Agriculture et biodiversité organisé par la Ligue de protection des oiseaux en octobre 2002, parrainé par les ministères de l'Écologie et de l'Agriculture et le Centre national pour l'aménagement des structures des exploitations agricoles, ne mentionne aucun agronome parmi les intervenants, et l'exposé sur «le travail du sol et la biodiversité en grandes cultures » est assuré conjointement par un agriculteur de l'Aisne et un représentant de l'Office national de la chasse et de la faune sauvage.

Pour revenir maintenant à la question de la nature de l'évolution des liens sociaux et des formes de solidarité autour des territoires ruraux, le constat de la pluralité des dispositifs environnementaux nuance fortement l'hypothèse émise au début de cette partie d'une remise en cause du mouvement de division du travail social. Si les agriculteurs, en effet, sont poussés à réintégrer dans leurs pratiques des éléments qu'ils avaient externalisés, ils le font sous l'injonction et le contrôle d'agents qui établissent avec eux, sur chacun des objectifs sectoriels, des relations contractuelles et contribuent à redéfinir leur(s) système(s) de droits et d'obligations au sein de la société. La prolifération des cahiers des charges - publics ou privés -, le plus souvent contractuels, que souscrivent les agriculteurs au titre de tel ou tel objectif environnemental - ou de cocktail d'objectifs, comme dans le cas des contrats territoriaux d'exploitation - participe de l'expansion des règles de droit restitutif et coopératif, indice de densité de leurs liens avec d'autres agents et groupes sociaux.

En s'enchevêtrant avec les anciennes, ces nouvelles règles sont source d'une certaine confusion dont la notion de multifonctionnalité tend à rendre compte. Cependant, c'est peut-être le maintien dans les analyses de la catégorie de pensée "agriculteur", forgée par le dispositif de modernisation (Rémy, 1987), qui pose problème. La redistribution différenciée, selon les territoires et les dispositifs environnementaux, des droits et obligations est sans doute porteuse d'un éclatement de cette catégorie, éclatement qui reste encore à concevoir et à qualifier. Le récent inventaire de la variété des termes aujourd'hui qualificatifs des agricultures (Pervanchon et Blouet, 2002) fournit peut-être la lexicologie dans laquelle se forgeront les catégories de la prochaine division du travail social dans le domaine.

Certains sociologues (Hervieu et Viard, 1996; Micoud, 1991) ont proposé de qualifier de "publicisation » le phénomène de multiplication des agents et groupes revendiquant de nouveaux droits de regard et d'usage sur les territoires ruraux. Ce terme rend assez bien compte à la fois de ce phénomène et du discours que tiennent ces derniers pour légitimer leurs demandes. Il est d'ailleurs symptomatique qu'en retour, ce soit au nom des droits de la propriété privée que se forgent les coalitions qui s'opposent à l'essor des dispositifs émergents d'administration environnementale des territoires, comme on a pu le voir avec le «Groupe des 9 » face à Natura 2000 (Alphandéry et Fortier, 2001). Cependant, il importe de rappeler que c'est aussi au titre de justifications d'intérêt public qu'ont été mis en place les droits d'usage prééminents de la catégorie des agriculteurs sur l'espace rural à l'époque de la modernisation. Il n'est pas certain que la redistribution des droits et obligations fonciers portés par les dispositifs émergents ne conduira pas à engendrer de nouvelles prééminences. J'en prendrai pour exemple la revendication par les conservatoires d'espaces naturels en France d'un réaménagement des règles du statut du fermage sur les territoires dont ils ont en charge la gestion.

\section{Conclusion}

Un autre des dangers de l'emploi du terme «nouveau » est la tentation de considérer des phénomènes émergents comme porteurs inéluctables de réorganisations radicales des systèmes précédents. Peut-être n'aije pas évité ce danger dans les propos qui précèdent. Il convient donc en conclusion d'apporter les réserves nécessaires pour ne pas tomber sous le coup de la critique d'amplifier les effets de tel ou tel dispositif dont la construction est loin d'être stabilisée.

On peut objecter en effet la force de résistance du dispositif de modernisation, aux structures bien établies et dont les ressorts d'innovation sont bien loin d'être 
épuisés. Les débats sur les organismes génétiquement modifiés en sont une illustration, où les environnementalistes se trouvent parfois à front renversé par rapport à certaines de leurs aspirations (comme la réduction des intrants ou les économies d'énergie). Sans doute cette situation tient-elle d'ailleurs au fait que les argumentaires des controverses dans ce domaine s'appuient, les uns et les autres, sur des justifications relevant des objectifs de la bio-politique. Quoi qu'il en soit, la pérennité des dispositifs des politiques environnementales n'est actuellement pas assurée, à commencer par leur soutien financier, et certains de leurs objectifs sont recyclables dans l'ancien. Il reste qu'il ne faut pas confondre les objectifs et ceux qui les portent, et il n'est pas certain que ces derniers seraient aussi facilement recyclables ou intégrables.

On peut également identifier d'autres mouvements qui remettent en cause les structures du dispositif de modernisation agricole dans les pays post-industriels sans pour autant intégrer explicitement les objectifs des politiques environnementales. Il s'agit ici bien évidemment des revendications liées à la libéralisation des échanges et au démantèlement des droits à subventions et à protection dont jouissent les agriculteurs. Les incertitudes des conséquences d'une telle dérégulation sont très fortes, et le déséquilibre des financements actuels entre la garantie et l'orientation de la politique agricole commune peut faire douter de la capacité de la seconde à compenser les effets de la disparition de la première. Néanmoins, on peut aussi faire l'hypothèse que l'affaiblissement d'une grande partie des agriculteurs lié au démantèlement de la protection peut accentuer le processus de redéfinition des droits et obligations des utilisateurs des territoires ruraux, et assurer davantage de place à tel ou tel des dispositifs émergents dans le domaine environnemental.

Ce qui peut changer dans cette perspective, c'est leur prise en charge par de nouveaux agents, davantage privés que publics ou associatifs, en particulier des firmes ayant choisi de faire des biens environnementaux des biens marchands. On ne serait là que dans la poursuite de La Grande Transformation capitaliste (Polanyi, 1983), dans l'extension du domaine de la production marchande, dans le processus que certains qualifient de "modernisation écologique» (Moll et Spaargaren, 2000). Des acquisitions foncières de la firme Vittel dans son périmètre de captage aux investissements des grandes firmes pharmaceutiques et de génie génétique dans des réserves de biodiversité en Amérique centrale, les prémisses d'une telle évolution ne manquent pas d'exister. Dans ce cadre, les agriculteurs ne deviendront pas les «jardiniers de la nature », mais les salariés des bio-industries.

\section{Références}

Alphandéry, P., Fortier, A., 2001. Can a territorial policy be based on science alone? The system for creating the Natura 2000 network in France, Sociologia Ruralis, 41, 3, 311-328.

Benoît, M., Papy, F., 1998. La place de l'agronomie dans la problématique environnementale, Les Dossiers de l'environnement de l'INRA, 17, 53-62.

Chombart de Lauwe, J., 1979. L'Aventure agricole de la France de 1945 à nos jours, Paris, PUF.

Datar, 2000. Schéma de services collectifs des espaces naturels et ruraux, Paris.

Deverre, C., 1995. Social implications of agro-environmental policy in France and Europe, Sociologia Ruralis, XXXV, 2, 227-247.

Deverre, C., Mormont, M., Soulard, C., 2002. La question de la nature et ses implications territoriales, in Perrier-Cornet $P$. (Ed.), Repenser les campagnes, L'Aube-DATAR, 217-237.

Dion, R., 1991. Essai sur la formation du paysage rural français, Paris, Flammarion.

Durkheim, E., 1930. De la division du travail social, Paris, PUF (1 $1^{\text {re }}$ édition 1893).

Foucault, M., 1975. Surveiller et punir, Paris, Gallimard.

Foucault, M., 1976. Histoire de la sexualité. I. La volonté de savoir, Paris, Gallimard.

Foucault, M., 1994. Dits et écrits. III. 1976-1979, Paris, Gallimard.

Gervais, M., Jollivet, M., Tavernier, Y., 1976. Histoire de la France rurale. Tome 4. La fin de la France paysanne. De 1914 à nos jours, Paris, Seuil.

Grafmeyer, Y., Joseph, I., 1990. L'École de Chicago. Naissance de l'écologie urbaine, Paris, Aubier-Montaigne.

Hervieu, B., Viard, J., 1996. Au bonheur des campagnes, L'Aube.

Larrère, C., Larrère, R., 1997. Du bon usage de la nature. Pour une philosophie de l'environnement, Paris, Aubier.

Lemaire, G., Meynard, J.M., 1998. Commentaires sur l'analyse de conjoncture concernant la place de l'agronomie dans la problématique environnementale, Les Dossiers de l'environnement de l'INRA, 17, 63-66.

Leopold, A., 1995. Almanach d'un comté des sables, Paris, Aubier.

Micoud, A. (Ed.), 1991. Des Hauts-lieux. La construction sociale de l'exemplarité, Paris, CNRS.

Moll, A.P.J., Spaargaren, G., 2000. Ecological Modernisation Theory in Debate: A Review, Environmental Politics, 9, 1, 17-50.

Papy, F., 2001. Pour une théorie du ménage des champs : 1'agronomie des territoires, C.R. Acad. Agric. Fr., 87, 4, 139-149.

Pervanchon F., Blouet A., 2002. Lexique des qualificatifs de l'agriculture, Le Courrier de l'environnement de l'INRA, 45, 117-137.

Pitte, J.-R., 1989. Histoire du paysage français, 2 tomes, Paris, Tallandier.

Polanyi, K., 1983. La Grande Transformation, Paris, Gallimard ( $1^{\text {re }}$ édition américaine 1944).

Rémy, J., 1987. La crise de professionnalisation en agriculture : les enjeux de la lutte pour le contrôle du titre d'agriculteur, Sociologie du travail, 4-87, 415-441.

Simmel, G., 1981. Sociologie et épistémologie, Paris, PUF (1 ${ }^{\text {re }}$ édition allemande 1900). 